\title{
Efficacy of low-dose thiopurine therapy for the induction of remission in steroid-dependent ulcerative colitis: Comparison with cytapheresis
}

\author{
Shuji Kochi*, Shotaro Nakamura, Takayuki Matsumoto \\ Department of Medicine and Clinical Science, Graduate School of Medical Sciences, Kyushu University, Fukuoka, Japan \\ Email: ${ }^{*}$ decorin@intmed2.med.kyushu-u.ac.jp
}

Received 14 November 2011; revised 23 December 2011; accepted 14 January 2012

\begin{abstract}
Background: The role of azathioprine (AZA) and 6mercaptopurine (6-MP) in the induction of remission in patients with ulcerative colitis (UC) remains unclear. Aims: To compare the efficacy and safety of low-dose thiopurine (AZA/6-MP) and cytapheresis (CAP) for the induction of remission in patients with steroiddependent UC. Patients and Methods: We reviewed the clinical course of 65 patients with steroid-dependent UC with moderate activity, who were treated with either low-dose AZA/6-MP (T-group, $n=38$ ) or with CAP (C-group, $n=27$ ). The efficacy and safety for the first 10 weeks after the start of the therapies were compared between the two groups. The cumulative probability curves of treatment failure were estimated by the Kaplan-Meier method. Clinical remission was defined as an ulcerative colitis activity index value of less than 150 without any other treatments. Results: Neither clinical characteristics, concomitant therapies, nor laboratory data (except for serum albumin levels) were different between the two groups. The remission rate at 10 weeks was not different between the two groups $(55.3 \%$ in the T-group and $70.4 \%$ in the C-group, $p=0.22$ in the intention-to-treat analysis). The frequencies of adverse events did not differ between the two groups $(p=0.12)$. The cumulative probability of treatment failure at 10 weeks was $44.7 \%$ for the T-group and $29.6 \%$ for the C-group ( $p=0.23$ ). Conclusions: Low-dose thiopurine therapy is an alternative candidate for the induction of remission in patients with steroid-dependent, moderate UC.
\end{abstract}

Keywords: Ulcerative Colitis, Azathioprine; 6-Mercaptopurine; Cytapheresis; Induction of Remission

\section{INTRODUCTION}

For patients with steroid-dependent or steroid-refractory

${ }^{*}$ Corresponding author. ulcerative colitis (UC), immunomodulatory agents, such as azathioprine (AZA), 6-mercaptopurine (6-MP), cyclosporine, or methotrexate, have been used for the induction and maintenance of clinical remission of the disease [1-10]. While thiopurines (AZA/6-MP) are the most commonly used agents to maintain clinical remission in patients with UC [1,2], the use of AZA/6-MP for inducing remission of active $\mathrm{UC}$ is still controversial [3-8]. In Western countries, a dose of AZA at $2.0-3.0 \mathrm{mg} /$ $\mathrm{kg} /$ day or of $6-\mathrm{MP}$ at $1.0-1.5 \mathrm{mg} / \mathrm{kg} / \mathrm{day}$ has been recommended to obtain a therapeutic efficacy $[2,9]$. However, a much lower dose of AZA ( $0.6-1.2 \mathrm{mg} / \mathrm{kg} /$ day $)$ is normally sufficient to maintain remission in Japanese patients with UC [10]. To date, the efficacy of such a low-dose of AZA/6-MP therapy for inducing remission in patients with UC has not been fully examined.

Cytapheresis (CAP) has also been reported to be a useful therapy for inducing clinical remission in patients with active UC, and is an alternative to systemic corticosteroid therapy [11-18]. CAP has been reported to reduce inflammation, promote the restoration of the intestinal mucosa, and suppress the production of proinflammatory cytokines by removing activated leukocytes from the peripheral blood in patients with active UC [1113], although the precise mechanisms underlying its therapeutic efficacy have not been fully elucidated.

While both AZA/6-MP and CAP are possible steroidsparing candidate treatments for patients with active UC, it has not yet been determined which is superior for the induction of remission. In the present study, we retrospectively compared the efficacy and safety of AZA/6MP and CAP in Japanese patients with steroid-dependent $\mathrm{UC}$.

\section{PATIENTS AND METHODS}

From April 2000 to October 2008, 126 Japanese patients with an established diagnosis of UC were treated with either low-dose thiopurines (AZA: $25-100 \mathrm{mg} / \mathrm{day}$ or 6-MP: 15 - $40 \mathrm{mg} /$ day; $\mathrm{n}=39)$ or with CAP $(\mathrm{n}=87)$ to 
induce clinical remission at our institution. The disease activity was assessed by the ulcerative colitis activity index (UCAI) [19], and a value of 150 or greater was regarded to indicate active disease. The inclusion criteria for the current study were as follows: 1) moderate activity, defined as an UCAI value between 150 and 220 prior to treatment [19]; 2) a dose of oral prednisolone $(\mathrm{PSL}) \leq 40 \mathrm{mg} /$ day prior to treatment; 3) no prior administration of AZA/6-MP; and 4) steroid-dependency with UCAI $\geq 150$ or greater after PSL had been tapered or discontinued. One patient who had been treated with AZA/6-MP and 60 patients who had been treated with CAP were excluded because they did not fulfill the criteria. Thirty-eight patients who were treated with thiopurines (AZA or 6-MP, T-group) and 27 patients treated with CAP (C-group) were therefore included in the present investigation.

CAP was carried out using either Adacolumn (JIMRO, Takasaki, Japan) for granulocyte and monocyte/macrophage adsorption apheresis (GCAP) [12] or Cellsorba EX (AsahiKaseiKurarayMedical, Tokyo, Japan) for leukocytapheresis (LCAP) [14]. Each patient in the C-group received CAP once a week up to a maximum of 10 apheresis sessions. Each session consisted of 60 minutes at a blood flow rate of between 30 and $50 \mathrm{~mL} / \mathrm{min}$. Continuous injection of anticoagulant, heparin or nafamostat mesilate was used to prevent coagulation of blood during extracorporeal circulation.

The demographic data for each patient was obtained by chart review. The data included gender, age, duration of the disease, the site of involvement, number of prior relapses, disease activity (UCAI), concomitant medications, and laboratory data (leukocyte counts, hemoglobin levels, platelet counts, serum albumin and C-reactive protein). During the course of treatment, PSL was tapered or discontinued when patients' symptoms were improved, while doses of oral 5-aminosalicylic acid (5-ASA, 1.5 $4.0 \mathrm{~g} /$ day; $\mathrm{n}=49$ ) and salazosulfapyridine (SASP, 3.0 $4.5 \mathrm{~g} /$ day; $\mathrm{n}=16$ ) were not changed. For the subsequent analyses, the doses of 5-ASA and SASP were adjusted by the molecular weights of each drug [20].

To assess the therapeutic effects of AZA/6-MP and $\mathrm{CAP}$, the UCAI was calculated just prior to each treatment and 4 and 10 weeks after the start of treatment. Clinical remission was defined as an UCAI value less than 150 without any other additional treatments. Treatment failure was defined by one of the following criteria: 1) discontinuation of AZA/6-MP or CAP due to adverse events; or 2) receiving additional treatments to induce remission. The safety and tolerability of AZA/6-MP and CAP were evaluated on the basis of the incidence and type of adverse events occurring during the observation period. All patients were regularly monitored for adverse events at each visit.
To assess the efficacy of the approaches, an intentionto-treat (ITT) statistical analysis was performed. The cumulative probability curves of treatment failure were calculated using the Kaplan-Meier method, and the differences between the groups were compared using the log rank test. Parametric data were expressed as the means $\pm \mathrm{SD}$, and compared between the groups using the Mann-Whitney U test. Nonparametric data were compared using the Fisher's exact probability test or Chi-squared test. A $p$ value $<0.05$ was considered to be statistically significant for each test.

\section{RESULTS}

\subsection{Demographic Data}

Table 1 compares the demographic data at baseline between the T-group and C-group. No significant differences were observed in gender, age, duration of the disease, site of involvement, UCAI, frequency and dose of PSL, adjusted dose of 5-ASA, or laboratory parameters between the groups. However, the serum albumin level was higher in the T-group than in the C-group ( $4.0 \pm 0.4$ vs. $3.7 \pm 0.5 \mathrm{~g} / \mathrm{dl}, p=0.006)$. The T-group also tended to show a lower number of prior relapses $(3.2 \pm 2.1$ vs. 3.9 \pm 1.5 times, $p=0.08)$ and a greater cumulative dose of PSL $(11.2 \pm 7.4$ vs. $8.2 \pm 6.7 \mathrm{~g}, p=0.06)$ than the C-group, although the differences were not statistically significant.

In the T-group, AZA was administered to 35 patients (92\%), and 6-MP to 3 patients (8\%). The doses of AZA and 6-MP were $54 \pm 14 \mathrm{mg} /$ day and $28 \pm 13 \mathrm{mg} /$ day, respectively. In the C-group, 20 patients (74\%) were treated with GCAP, and 7 patients (26\%) with LCAP.

\subsection{Clinical Outcome of AZA/6-MP and CAP Treatment}

As shown in Table 2, the rates of inducing remission in the T-group and C-group at 4 weeks were $50.0 \%(19 / 38)$ and $55.6 \%(15 / 27)$, respectively $(p=0.66)$. At 10 weeks, the rates were $55.3 \%(21 / 38)$ and $70.4 \%(19 / 27)$, respectively $(p=0.22)$, in the T- and C-groups. Treatment failure was observed in 17 of 38 patients in the T-group and 8 of 27 patients in the C-group. Figure 1 demonstrates the cumulative probability curves of treatment failure in the two groups. The cumulative probabilities of treatment failure at 10 weeks for the T-group and C-group were $44.7 \%$ and $29.6 \%$, respectively $(p=0.23)$.

In 12 of the 17 patients with treatment failure in the T-group, the clinical symptoms were either unchanged or deteriorated, and the patients achieved clinical remission after additional treatment by increasing the dose of PSL $(\mathrm{n}=9)$, or by performing CAP $(\mathrm{n}=3)$. The remaining 5 patients discontinued AZA due to adverse events, and a 
Table 1. Comparison of the baseline characteristics of patients in the T-group and C-group.

\begin{tabular}{|c|c|c|c|}
\hline Clinical variables & $\begin{array}{l}\text { T-group } \\
(\mathrm{n}=38)\end{array}$ & $\begin{array}{l}\text { C-group } \\
(\mathrm{n}=27)\end{array}$ & $p$ value \\
\hline Gender (female/male) & $30 / 8$ & $20 / 7$ & 0.65 \\
\hline Age (years) & $37.0 \pm 14.1$ & $38.3 \pm 14.1$ & 0.69 \\
\hline Duration of the disease (years) & $5.4 \pm 4.9$ & $6.7 \pm 4.9$ & 0.19 \\
\hline \multicolumn{4}{|l|}{ Site of involvement } \\
\hline Pancolitis & 26 & 14 & 0.18 \\
\hline Left-sided colitis & 12 & 13 & \\
\hline Number of prior relapses (times) & $3.2 \pm 2.1$ & $3.9 \pm 1.5$ & 0.08 \\
\hline Disease activity (UCAI) & $188 \pm 16$ & $194 \pm 12$ & 0.17 \\
\hline Cumulative dose of PSL (g) & $11.2 \pm 7.4$ & $8.2 \pm 6.7$ & 0.06 \\
\hline Concomitant use of PSL & 36 & 23 & 0.22 \\
\hline Dose of PSL at entry (mg/day) & $16.4 \pm 10.9$ & $17.4 \pm 12.8$ & 0.82 \\
\hline Dose of 5-ASA (mg/day) & $2108 \pm 589$ & $2241 \pm 399$ & 0.11 \\
\hline Dose of AZA (mg/day; $\mathrm{n}=35)$ & $53.6 \pm 13.8$ & - & \\
\hline Dose of 6-MP $(\mathrm{mg} /$ day; $\mathrm{n}=3)$ & $28.3 \pm 12.6$ & - & \\
\hline \multicolumn{4}{|l|}{ Laboratory data } \\
\hline Leukocyte counts $(/ \mu 1)$ & $8968 \pm 2694$ & $9366 \pm 3869$ & 0.80 \\
\hline Hemoglobin levels (g/dl) & $12.1 \pm 1.7$ & $11.7 \pm 2.1$ & 0.46 \\
\hline Platelet counts $(\times 104 / \mu 1)$ & $32.6 \pm 7.2$ & $30.6 \pm 7.2$ & 0.26 \\
\hline Serum albumin $(\mathrm{g} / \mathrm{dl})$ & $4.0 \pm 0.4$ & $3.7 \pm 0.5$ & 0.006 \\
\hline C-reactive protein $(\mathrm{mg} / \mathrm{dl})$ & $0.6 \pm 0.7$ & $1.3 \pm 2.2$ & 0.50 \\
\hline
\end{tabular}

T-group: thiopurines-group; C-group: cytapheresis-group; UCAI: ulcerative colitis activity index; PSL: prednisolone; 5-ASA: 5-aminosalicylic acid; AZA: azathioprine; 6-MP: 6-mercaptopurine.

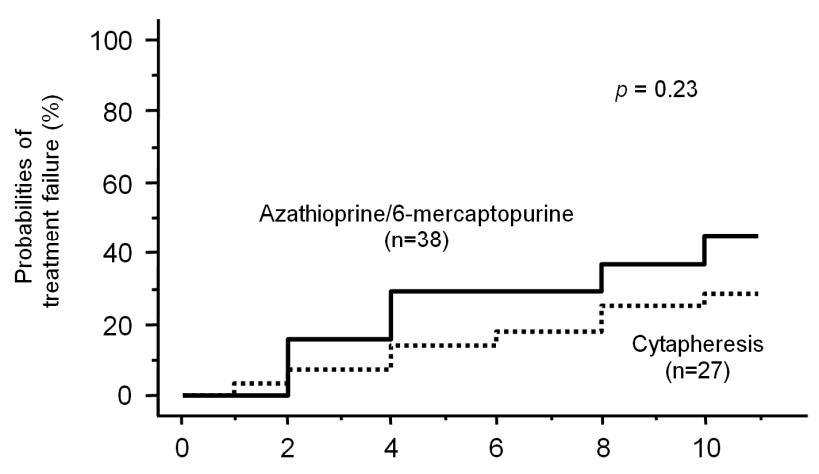

Figure 1. The cumulative probability curves of treatment failure in patients with steroid-dependent ulcerative colitis, as stratified by treatment group (thiopurines-group and cytapheresis-group; $p=0.23$ : Log rank test).

Subsequent increase in the dose of PSL resulted in clinical remission in these patients. In all 8 patients who experienced treatment failure in the C-group, the clinical symptoms were either unchanged or deteriorated. Seven of these 8 patients achieved clinical remission after additional treatments; 5 patients achieved remission by starting PSL or increasing the dose of PSL together with CAP, one patient by starting AZA after completing 10 sessions of CAP, and one patient by starting cyclosporine and increasing the dose of PSL after discontinuing CAP. The remaining one patient underwent an emergency colectomy after the first session of CAP.

\subsection{Adverse Events Attributable to AZA/6-MP or CAP}

As shown in Table 2, adverse events were observed in 5 of 38 patients (13.2\%) in the T-group and 8 of 27 patients $(29.6 \%)$ in the C-group $(p=0.12)$. In the Tgroup, adverse events included acute pancreatitis, erythema nodosum, fever, arthralgia, myalgia, and liver damage, which occurred during a period from 21 to 30 days after starting AZA. In all 5 patients, the adverse events spontaneously disappeared after the discontinuation of AZA. In the C-group, adverse events including transient headache, fatigue, and nausea were observed only during the sessions of CAP. No patients discontinued CAP, since these adverse events were considered to be tolerable.

\section{DISCUSSION}

Previous studies have reported that AZA at a dose of 2 $2.5 \mathrm{mg} / \mathrm{kg} /$ day [3-6] and 6-MP at a dose of $50-150$ $\mathrm{mg} /$ day [7] or $1.5 \mathrm{mg} / \mathrm{kg} /$ day [8] were effective in inducing clinical remission in active [4-6] or steroid-dependent $[3,7,8]$ UC, while others have failed to demonstrate such an efficacy [21,22]. A systematic review of 30 uncontrolled studies showed that the mean efficacy rate of AZA/6-MP was $65 \%$ for the induction of the remission in active UC patients [2]. However, a meta-analysis of 4 controlled 
studies revealed no significant benefit of AZA/6-MP for inducing remission in comparison with placebo or 5-ASA (odds ratio; 1.59, 95\% confidence interval; 0.59 - 4.29) [2].

The efficacy of CAP has been shown not only for steroidnaïve patients who had never received corticosteroids $[11,13]$, but also for steroid-dependent [11,15-18] and steroid-refractory $[15,17]$ patients. Previous studies have demonstrated that $58 \%-90 \%$ of patients with steroiddependent UC who had concomitant use of PSL achieved improvement or clinical remission following a course of
CAP [11,15-18]. CAP has also been shown to have a steroid sparing effect $[11,12,15,16]$, as has been seen for AZA/6-MP therapy [3,4,6-8].

Our present investigation is the first study to examine the efficacy of low-dose AZA/6-MP for inducing remission, and which compared the efficacy of AZA/6-MP and CAP in patients with steroid-dependent UC. We found the efficacy of low-dose AZA/6-MP for the induction of remission in steroid-dependent, moderate $\mathrm{UC}$ to be $55.3 \%$ at 10 weeks, and this observed efficacy was not statistically different from that of CAP (Table 3).

Table 2. Adverse events in patients with ulcerative colitis after treatment with azathioprine/6-mercarptopurine or cytapheresis.

\begin{tabular}{|c|c|c|c|c|}
\hline Therapy & Age (years) & Gender & Adverse events & Action \\
\hline \multicolumn{5}{|l|}{ T-group } \\
\hline AZA & 19 & Female & Acute pancreatitis & Withdrawal \\
\hline AZA & 38 & Female & Erythema nodosum/arthralgia & Withdrawal \\
\hline AZA & 46 & Female & Erythema nodosum/fever/liver damage ${ }^{*}$ & Withdrawal \\
\hline AZA & 54 & Female & Fever/liver damage $^{*}$ & Withdrawal \\
\hline AZA & 71 & Male & Myalgia of the lower legs & Withdrawal \\
\hline \multicolumn{5}{|l|}{ C-group } \\
\hline GCAP & 31 & Female & Headache & CAP continued, medication \\
\hline GCAP & 32 & Female & Fatigue & CAP continued \\
\hline GCAP & 33 & Female & Nausea/fatigue & CAP continued, medication \\
\hline GCAP & 37 & Male & Headache & CAP continued, medication \\
\hline GCAP & 65 & Male & Headache & CAP continued, medication \\
\hline LCAP & 25 & Female & Headache & CAP continued, medication \\
\hline LCAP & 32 & Female & Headache/fatigue & CAP continued, medication \\
\hline LCAP & 40 & Female & Headache/fatigue & CAP continued, medication \\
\hline
\end{tabular}

T-group: thiopurines-group; AZA: azathioprine; C-group: cytapheresis-group; GCAP: granulocyte and monocyte/macrophage adsorption apheresis; LCAP: leukocytapheresis. " Liver damage is defined as an elevation of liver enzymes, such as aspartate aminotransferase and alanine aminotransferase to more than 2 times the upper limit of normal.

Table 3. Outcomes of azathioprine/6-mercaptopurine and cytapheresis therapy.

\begin{tabular}{cccccc}
\hline & 4 weeks & & \\
\hline Remission rate & $\begin{array}{c}\text { T-group } \\
(\mathrm{n}=38)\end{array}$ & $\begin{array}{l}\text { C-group } \\
(\mathrm{n}=27)\end{array}$ & $p$ value & $\begin{array}{l}\text { T-group } \\
(\mathrm{n}=38)\end{array}$ & $\begin{array}{l}\mathrm{C} \text {-group } \\
(\mathrm{n}=27)\end{array}$ \\
\hline Intention-to-treat analysis & $19(50.0 \%)$ & $15(55.6 \%)$ & 0.66 & $21(55.3 \%)$ & $19(70.4 \%)$ \\
\hline
\end{tabular}

T-group: thiopurines-group; C-group: cytapheresis-group. 
The probabilities of treatment failure at 10 weeks, as well as the frequencies of adverse events, were not signifycantly different between the two groups. These results suggest that AZA at $25-100 \mathrm{mg} /$ day or 6-MP at $15-40$ $\mathrm{mg} /$ day, which are much lower than those used in Western countries [3-8], may be applicable for the induction of remission in Japanese patients with steroid-dependent UC. Since it has been demonstrated that AZA and 6-MP usually have a slow onset of action, with a mean time from initiation to the clinical response of 3.1 months [23], these drugs have not been suggested as a monotherapy for acute relapses of UC. In our study, however, AZA/6MP actually induced clinical remission within a relatively short time. Of all 21 patients who achieved remission by AZA/6-MP by 10 weeks after initiating treatment, 19 patients achieved remission within 4 weeks. The efficacy rates of AZA/6-MP (55.3\%) and CAP (70.4\%) in our study are similar to those in previous reports (AZA/ 6-MP 53\% - 92\%; CAP 58\% - 90\%), which also included subjects who received concomitant corticosteroid therapy [3,6-8,11,12,15-18].

The benefits of CAP for active UC are not only its efficacy in inducing clinical remission, but also its safety and tolerability. Adverse events attributable to CAP including dizziness, nausea, headache, flushing and fever have been observed in $5 \%-27 \%$ of patients [24]. However, no serious adverse events have been reported during or after CAP, and almost all patients can complete the course of therapy. However, a serious problem is that CAP is more expensive than other therapies for UC $[24,25]$. In addition, CAP requires the priming of the CAP system by saline containing anticoagulant, venous access of bilateral antecubital veins, bed rest for at least an hour, and frequent hospital visits in the case of outpatients. Furthermore, CAP is occasionally complicated withinfectious problems, such as pneumonia or catheter-induced septicemia [18]. In consideration of the above, CAP may inflict both physical and psychological distress on UC patients.

For active UC, AZA/6-MP is less expensive and more convenient than CAP. However, both AZA and 6-MP can induce serious adverse events, with an incidence ranging from $5 \%$ up to $40 \%$, in both a dose-dependent and -independent manner [26]. Previous studies have reported that patients treated with AZA/6-MP experienced severe adverse events when compared to patients treated with CAP, including events such as pancytopenia, acute pancreatitis, fever, headache, rash, arthralgia, myalgia, liver damage, gastrointestinal disturbances, and infections [26], most of which led to the discontinuation of the drugs. Therefore, patients treated with AZA/6-MP need careful and regular supervision to monitor the development and incidence of adverse events.

Our present study has a number of limitations. First, with regard to the data collection, the retrospective and single-center nature of the study, and the small number of subjects, are potential sources of selection bias. Second, each treatment group consisted of heterogeneous modalities; the C-group included both GCAP and LCAP, and the T-group included both AZA and 6-MP. Finally, we did not evaluate the mucosal healing by colonoscopy. These limitations may have hampered the precise analysis of the effects of the treatments.

In conclusion, our retrospective study indicated that low-dose AZA/6-MP therapy appears to be an effective, inexpensive and convenient therapeutic option for the induction of clinical remission in patients with steroiddependent, moderate UC. While the efficacy for the induction of remission was not statistically different between low-dose AZA/6-MP therapy and CAP in our study, the number of cases that discontinue treatment due to adverse events was higher in the T-group than in the C-group. Prospectively designed studies with a large number of subjects are needed to confirm the efficacy and safety of the treatment with AZA/6-MP.

\section{REFERENCES}

[1] Timmer, A., McDonald, J.W. and Macdonald, J.K. (2007) Azathioprine and 6-mercaptopurine for maintenance of remission in ulcerative colitis. Cochrane Database of Systematic Reviews, 1, CD000478.

[2] Gisbert, J.P., Linares, P.M., McNicholl, A.G., et al. (2009) Meta-analysis: The efficacy of azathioprine and mercaptopurine in ulcerative colitis. Aliment Pharmacol \& Therapeutics, 30, 126-137. doi:10.1111/j.1365-2036.2009.04023.x

[3] Ardizzone, S., Maconi, G., Russo, A., et al. (2006) Randomised controlled trial of azathioprine and 5-aminosalicylic acid for treatment of steroid dependent ulcerative colitis. Gut, 55, 47-53. doi:10.1136/gut.2005.068809

[4] Kirk, A.P. and Lennard-Jones, J.E. (1982) Controlled trial of azathioprine in chronic ulcerative colitis. British Medicine Journal, 284, 1291-1292. doi:10.1136/bmj.284.6325.1291

[5] Caprilli, R., Carratù, R. and Babbini, M. (1975) Doubleblind comparison of the effectiveness of azathioprine and sulfasalazine in idiopathic proctocolitis. Preliminary report. The American Journal of Digestive Diseases, 20, 115-120. doi:10.1007/BF01072336

[6] Orth, T., Peters, M., Schlaak, J.F., et al. (2000) Mycophenolate mofetil versus azathioprine in patients with chronic active ulcerative colitis: A 12-month pilot study. The American Journal of Gastroenterology, 95, 12011207. doi:10.1111/j.1572-0241.2000.02010.x

[7] Adler, D.J. and Korelitz, B.I. (1990) The therapeutic efficacy of 6-mercaptopurine in refractory ulcerative colitis. The American Journal of Gastroenterology, 85, 717-722.

[8] Maté-Jiménez, J., Hermida, C., Cantero-Perona, J., et al. (2000) 6-Mercaptopurine or methotrexate added to pred- 
nisone induces and maintains remission in steroid-dependent inflammatory bowel disease. European Journal of Gastroenterology and Hepatology, 12, 1227-1233. doi:10.1097/00042737-200012110-00010

[9] Lichtenstein, G.R., Abreu, M.T., Cohen, R., et al. (2006) American Gastroenterological Association Institute technical review on corticosteroids, immunomodulators, and infliximab in inflammatory bowel disease. Gastroenterology, 130, 940-987. doi:10.1053/j.gastro.2006.01.048

[10] Hibi, T., Naganuma, M., Kitahora, T., et al. (2003) Lowdose azathioprine is effective and safe for maintenance of remission in patients with ulcerative colitis. Journal of Gastrotenterology, 38, 740-746. doi:10.1007/s00535-003-1139-2

[11] Tanaka, T., Okanobu, H., Yoshimi, S., et al. (2008) In patients with ulcerative colitis, adsorptive depletion of granulocytes and monocytes impacts mucosal level of neutrophils and clinically is most effective in steroid naïve patients. Digestive and Liver Disease, 40, 731-736. doi:10.1016/j.dld.2008.02.012

[12] Shimoyama, T., Sawada, K., Hiwatashi, N., et al. (2001) Safety and efficacy of granulocyte and monocyte adsorption apheresis in patients with active ulcerative colitis: A multicenter study. Journal of Clinical Apheresis, 16, 1-9. doi:10.1002/jca.1000

[13] Suzuki, Y., Yoshimura, N., Saniabadi, A.R., et al. (2004) Selective granulocyte and monocyte adsorptive apheresis as a first-line treatment for steroid naïve patients with active ulcerative colitis: A prospective uncontrolled study. Digestive Diseases and Science, 49, 565-571. doi:10.1023/B:DDAS.0000026299.43792.ae

[14] Sawada, K., Ohnishi, K., Fukui, S., et al. (1995) Leukocytapheresis therapy, performed with leukocyte removal filter, for inflammatory bowel disease. Journal of Gastroenterology, 30, 322-329. doi:10.1007/BF02347507

[15] Naganuma, M., Funakoshi, S., Sakuraba, A., et al. (2004) Granulocytapheresis is useful as an alternative therapy in patients with steroid-refractory or -dependent ulcerative colitis. Inflammmtory Bowel Diseases, 10, 251-257. doi:10.1097/00054725-200405000-00012

[16] Hanai, H., Watanabe, F., Yamada, M., et al. (2004) Adsorptive granulocyte and monocyte apheresis versus prednisolone in patients with corticosteroid-dependent moderately severe ulcerative colitis. Digestion, 70, 36-44.

\section{doi:10.1159/000080079}

[17] Takemoto, K., Kato, J., Kuriyama, M., et al. (2007) Predictive factors of efficacy of leukocytapheresis for steroid-resistant ulcerative colitis patients. Digestive and Liver Disease, 39, 422-429. doi:10.1016/j.dld.2007.01.010

[18] Domènech, E., Hinojosa, J., Esteve-Comas, M., et al. (2004) Granulocyteaphaeresis in steroid-dependent inflammatory bowel disease: A prospective, open, pilot study. Alimentary Pharmacology \& Therapeutics, 20, 1347-1352. doi:10.1111/j.1365-2036.2004.02288.X

[19] Seo, M., Okada, M., Yao, T., et al. (1992) An index of disease activity in patients with ulcerative colitis. The American Journal of Gastroenterology, 87, 971-976.

[20] Sandborn, W.J. and Hanauer, S.B. (2003) Systematic review: The pharmacokinetic profiles of oral mesalazine formulations and mesalazine pro-drugs used in the management of ulcerative colitis. Alimentary Pharmacology \& Therapeutics, 17, 29-42. doi:10.1046/j.1365-2036.2003.01408.x

[21] Jewell, D.P. and Truelove, S.C. (1974) Azathioprine in ulcerative colitis: Final report on controlled therapeutic trial. British Medicine Journal, 4, 627-630. doi:10.1136/bmj.4.5945.627

[22] Sood, A., Midha, V., Sood, N., et al. (2000) Role of azathioprine in severe ulcerative colitis: One-year, placebocontrolled, randomized trial. Indian Journal of Gastroenterology, 19, 14-16.

[23] Present, D.H., Korelitz, B.I., Wisch, N., et al. (1980) Treatment of Crohn's disease with 6-mercaptopurine. A longterm, randomized, double-blind study. The New England Journal of Medicine, 302, 981-987. doi:10.1056/NEJM198005013021801

[24] Yamamoto, T., Umegae, S. and Matsumoto, K. (2006) Safety and clinical efficacy of granulocyte and monocyte adsorptive apheresis therapy for ulcerative colitis. World Journal of Gastroenterology, 12, 520-525.

[25] Panés, J., Guilera, M., Ginard, D., et al. (2007) Treatment cost of ulcerative colitis is apheresis with Adacolumn cost-effective? Digestive and Liver Disease, 39, 617-625.

[26] Teml, A., Schaeffeler, E., Herrlinger, K.R., et al. (2007) Thiopurine treatment in inflammatory bowel disease: Clinical pharmacology and implication of pharmacogenetically guided dosing. Clinical Pharmacokinetics, 46, 187208. doi:10.2165/00003088-200746030-00001 